\title{
Benign and Malicious Envy Scale (BeMaS): Evidências de Validade para Adultos Brasileiros
}

\author{
Benign and Malicious Envy Scale (BeMaS): Evidence of Validity for Brazilian \\ Adults
}

\author{
Evandro Morais Peixoto ${ }^{1}$, Amanda Rizzieri Romano², Marcus Vinícius Silva², \\ Bruno Cesar de Almeida ${ }^{3}$ e Carolina Rosa Campos ${ }^{4}$
}

\begin{abstract}
Resumo
A inveja é um fenômeno psicológico comum às diferentes culturas. O objetivo foi estimar evidências de validade da Benign and Malicious Envy Scale (BeMaS), instrumento que propõem a mensuração de duas dimensões da inveja: benigna e maliciosa, em adultos brasileiros. Participaram 409 adultos não clínicos de ambos os sexos $(\mathrm{M}=28.5 \pm 10.4 ; 74.3 \%$ mulheres $)$. Foram obtidas evidências de validade baseadas na estrutura interna, na relação com outras variáveis (ansiedade, depressão, distresse psicológico global, e tríade sombria da personalidade). A Análise Fatorial Confirmatória sugeriu adequação da estrutura composta por dois fatores (CFI=.954; TLI=.934; RMSEA=.087). Adicionalmente, obteve-se indicadores de invariância do modelo de medida entre adultos e adolescentes (amostra recuperada de estudo prévio). Por fim, verificou-se relações com variáveis externas coerentes com as hipóteses teóricas. Os resultados sugerem adequação da BeMaS para mensuração da inveja em adultos brasileiros e demonstra potencialidade do instrumento para ser empregado em futuras pesquisas.
\end{abstract}

Palavras-chave: comparação social, tríade sombria, personalidade, psicometria, validade

\begin{abstract}
Envy is a psychological phenomenon common to different cultures. This study aimed at estimating validity evidence of the Benign and Malicious Envy Scale (BeMaS), designed to measure two dimensions of envy: benign and malicious, in Brazilian adults. The sample was composed of 409 non-clinical adults of both sexes $(\mathrm{M}=28.5 \pm 10.4 ; 74.3 \%$ women $)$. Validity evidence was estimated based on the internal structure and on the relation to other variables (anxiety, depression, global psychological distress, and dark triad of personality). Confirmatory Factor Analysis demonstrated adequacy of the two-factor structure (CFI=.954; TLI=.934; RMSEA=.087). Evidence of measurement invariance between adults and adolescents (a sample recovered from previous studies) was obtained. Correlations with external variables consistent with the theoretical hypotheses were observed. The results suggest the adequacy of the BeMaS to measure envy in Brazilian adults and its potential to be used in future research.
\end{abstract}

Keywords: social comparison, dark triad, personality, psychometrics, validity

\footnotetext{
${ }^{1}$ Psicólogo, Doutor em Psicologia como profissão e ciência. Docente do Programa de Pós-Graduação em Psicologia da Universidade São Francisco - USF, Rua Waldemar César da Silveira, 105 - Jardim Cura D'ars, Campinas - SP, 13045-510, Brasil. Tel.: +55 019 3779-3396. E-mail: evandro.peixoto@usf.edu.br

2 Psicólogo. Discente do Programa de Pós Graduação em Psicologia da Universidade São Francisco - USF, Brasil. E-mail: amanda.romano@mail.usf.edu.br; marcus.vinicius@mail.usf.edu.br

${ }^{3}$ Psicólogo. Universidade São Francisco - USF, Brasil. E-mail: bc.brunoalmeida@ gmail.com

${ }^{4}$ Psicóloga, Doutora em Psicologia como profissão e ciência. Docente Adjunta do Curso de Psicologia da Universidade Federal do Triângulo Mineiro - UFTM, Brasil. Email: carolinarosacampos@gmail.com

Revista Iberoamericana de Diagnóstico y Evaluación - e Avaliação Psicológica. RIDEP · No58 · Vol.1 · 67-77 · 2021

ISSN: 1135-3848 print /2183-6051online
} 


\section{Introdução}

A inveja, independentemente da cultura, é uma emoção comumente vivenciada pelas pessoas. Caracteriza-se como um sentimento desagradável que emerge quando os indivíduos se comparam de maneira desfavorável em relação às outras pessoas, bem como, implica sentimentos despertados quando se deseja algo que é de posse do outro (Lange \& Crusius, 2015; Smith \& Kim, 2007). Dessa forma, o construto da inveja envolve diversas tendências afetivas, cognitivas e motivacionais (Milfont \& Gouveia, 2009).

Estudos iniciais sobre a inveja compreendemna como um fenômeno episódico e negativo, no qual o indivíduo direcionava reações hostis às pessoas ou objetos invejados. Entretanto, ao considerar as diferenças individuais, pesquisas evidenciam que a inveja está associada a características disposicionais, definidas como unitárias e explicadas por dois aspectos principais: a) inferioridade, desencadeada pela interpretação da comparação social ascendente de maneira negativa e b) senso de injustiça subjetivo (Crusius, Gonzalez, Lange, \& Cohen-Charash, 2019; Van de Ven, 2016). Sendo assim, a partir de estudos recentes, nota-se que o construto vem sendo considerado em uma perspectiva bidimensional em que duas formas de lidar com as emoções podem surgir ao se realizar comparações sociais: depreciar aquele que considera superior ou busca pela melhoria da própria posição, reações essas que emergem de situações de comparações sociais, consideradas importantes e que ameaçam a autoimagem do indivíduo (Kwiatkowska et al., 2020).

Independente das particularidades linguísticas, é possível identificar diferenciação etimológica dos termos para referenciar-se a uma inveja considerada motivacional daquela inveja considerada negativa. Isso porque, a inveja pode ser compreendida de forma dualística, sob uma perspectiva de inveja benigna e maliciosa (Parrott, 1991; Smith \& Kim, 2007). Neste sentido, considerando a inveja benigna, pode-se dizer que o teor motivacional da inveja faz com que o desejo de obter o que a pessoa invejada tem aumente, entretanto, sem a presença da hostilidade, característica predominante na inveja maliciosa.
Isso significa que ambos os tipos de inveja refletem maneiras de se atingir o objetivo de equiparar-se ao indivíduo ou objeto invejado, nivelando a diferença entre o eu e o padrão superior de comparação. No caso da inveja benigna pode haver o impulsionamento do sujeito, o qual pode buscar tornar-se tão bem sucedido quanto o outro, enquanto o sujeito que experimenta a inveja maliciosa utiliza outros recursos para nivelar-se, como a diminuição ou difamação da vantagem alheia, gerando motivação para prejudicar a pessoa invejada (Lange \& Crusius, 2015).

Nos seus diferentes aspectos, a inveja proporciona a vivência de sentimentos desagradáveis, as quais estão ligadas ao tipo de inveja sentida, produzindo-se, assim, comportamentos e motivações distintas. A partir dessas duas facetas, a inveja benigna surge quando o indivíduo compreende a superioridade do outro como algo que foi merecido, diferentemente da situação que resulta em inveja maliciosa, no qual a reação surge a partir da interpretação de que a vantagem não é correspondente ao invejado. Vale ressaltar, que em ambas as experiências, há um nível elevado de afetos negativos e frustração (Van de Ven et al., 2009).

A natureza hostil que circunda o construto inveja, torna o processo de medi-la complexo, pois os indivíduos tendem a ocultar, negar ou silenciar a experiência vivenciada, pelo fato de ser considerada socialmente indesejável. Entretanto, essa concepção ampliada do conceito, que considera também características que impulsionam o sujeito, desvinculando-se do aspecto excepcionalmente negativo, possibilita o acesso a maior extensão deste construto psicológico, potencializando o processo de mensuração. Dessa forma, Lange e Crusius (2015), considerando os aspectos teóricos da inveja disposicional, desenvolveram na Alemanha a Benign and Malicious Envy Scale (BeMaS).

A BeMaS é um instrumento de autorrelato, com o intuito de aferir as duas formas de inveja, por meio de 10 itens com chave de resposta do tipo Likert de sete pontos, divididos em cinco itens para cada expressão do construto. Ambos os fatores demonstraram boa consistência interna, $\alpha($ benigna $)=.79-.90, \alpha($ maliciosa $)=.83-.91 . \mathrm{A}$ BeMaS tem sido adaptada para diferentes culturas e 
países (Estados Unidos, Bósnia, Bulgária, China, Espanha, Estônia, Holanda, Hungria, Japão, Indonésia, Irã, Portugal, Polônio Rússia, Sérvia, Turquia, Ucrânia e Vietnã) e estes estudos corroboram com a expectativa teórica ao apresentar evidências de validade no que se refere a uma estrutura interna composta por dois fatores e bons indicadores de precisão ao instrumento (NavarroCarrilo et al., 2017; Kwiatkowska et al., 2020; Peixoto et al., no prelo; Sawada \& Fujii, 2016).

A BeMaS foi adaptada transculturalmente para o português brasileiro por Peixoto et al. (no prelo), que avaliaram as evidências de validade do instrumento em uma amostra composta por 248 adolescentes provenientes da região sudeste do Brasil. A avaliação da estrutura interna, realizada por meio de Análise Fatorial Exploratória (AFE), demonstrou adequação da matriz de correlação, com os índices Kaiser-Meyer-Olkin (KMO=.789) e Teste de Esfericidade de Bartlett significativos, bem como a adequação da retenção de dois fatores, correspondentes a uma estrutura bidimensional da inveja (benigna e maliciosa). Adicionalmente, os autores verificam índices de precisão satisfatórios para ambos fatores do instrumento com $\alpha$ iguais .72 e .82 , respectivamente e $\omega$ iguais .76 e .82 , respectivamente.

Peixoto et al (no prelo) também verificaram evidências de validade baseada na relação com outras variáveis. Para tanto, os autores estimaram índices de correlações de Pearson entre os indicadores de inveja com depressão, ansiedade, estresse, distresse psicológico global e satisfação com a vida. De acordo com os dados obtidos, observou-se que a inveja maliciosa apresenta correlação positiva de magnitude moderada com as variáveis ansiedade ( $r=.39)$, depressão $(r=.36)$ e distresse global $(r=.38)$. Além disso, verificouse correlações de baixa magnitude entre a inveja maliciosa com as variáveis estresse $(r=.24) \mathrm{e}$ satisfação com a vida ( $r=-.25)$. Desse modo, é possível inferir que existe uma tendência de que indivíduos com elevado nível de inveja maliciosa apresentem diminuição dos índices de saúde mental e na satisfação com a vida. Entretanto, obteve-se uma correlação positiva de baixa magnitude entre a inveja benigna e satisfação com a vida $(r=.20)$ e ausência de associação com os indicadores de depressão ( $r=-.08)$, estresse $(r=-$
.01 ), ansiedade ( $r=-.09$ ), e distresse global ( $r=-$ $.07)$, o que sugere que a vivência de níveis inveja benigna não está associado a prejuízos nestes indicadores de saúde mental.

A possibilidade de operacionalização do modelo bidimensional da inveja, a estabilidade da medida observada em diferentes culturas e a aplicabilidade do instrumento com pesquisas que associam a inveja a outros fenômenos psicológicos como autoeficácia, motivação para metas (NavarroCarrilo, 2017; Sawada \& Fujii, 2016), performance esportiva (Lange \& Crusius, 2015) e tríade sombria (Lange et al., 2018) sugerem que a BeMaS é um instrumento de grande valia para a compreensão do construto. No que diz respeito a versão brasileira da BeMaS, ainda são necessários novos estudos referentes às suas propriedades psicométricas, haja vista que em estudo inicial a amostra foi composta apenas por adolescentes. Considerando a lacuna existente, pretende-se nesta pesquisa ampliar a coleta de dados com enfoque no público adulto, para acúmulo de evidências científicas em relação às potencialidades do instrumento para esta população.

\section{Objetivos do estudo}

Com base no exposto, a presente pesquisa tem como principal objetivo obter evidências de validade para a BeMaS, com base na estrutura interna e relação com variáveis externas, a saber: a depressão, ansiedade, distresse psicológico global, maquiavelismo, psicopatia e narcisismo, em uma amostra composta por adultos. Adicionalmente, a amostra de adolescentes proveniente do estudo Peixoto et al. (no prelo) será recuperada para avaliação da invariância do modelo de medida (Configuracional, métrico e escalar) entre grupos estabelecidos em função destas etapas do desenvolvimento.

$\mathrm{O}$ estudo se baseia nas hipóteses de confirmação do modelo bidimensional da inveja neste novo estrato populacional, bem como na invariância do modelo de medida entre as diferentes faixas-etárias (adolescentes e adultos). Espera-se também, por meio da busca de novas evidências de validade com base na relação com variáveis externas, dos tipos convergente e discriminante, encontrar correlações positivas entre inveja maliciosa, depressão, ansiedade, distresse psicológico global (Peixoto et al., no prelo), e 
também com maquiavelismo, narcisismo e psicopatia. Adicionalmente, espera-se encontrar correlações positivas entre inveja benigna e os indicadores da tríade sombria da personalidade, e ausência de associação desta expressão da inveja com as variáveis depressão, ansiedade e distresse psicológico global. Estas hipóteses foram criadas sob a perspectiva de que, embora a inveja benigna esteja associada a ações direcionadas ao crescimento pessoal e a fenômenos psicológicos positivos, pode ainda haver relação com determinadas características psicopatológicas, uma vez que, de modo mais amplo, este componente da inveja também se caracteriza como um sentimento de inferioridade que emerge a partir de comparações sociais e visa o emprego de estratégias para superar essas limitações (Lange \& Crusius, 2015; Lange, Paulhus, \& Crusius, 2018).

\section{Método}

\section{Participantes}

A amostra principal foi composta por 409 adultos, de ambos os sexos $(74.3 \%$ do sexo feminino), com idades entre 18 e 67 anos $(M=$ $28.5 \pm 10.4$ ), provenientes de diferentes regiões do Brasil (85.6\% Sudeste, 6.6\% Sul, 3.4\% Nordeste, $2.9 \%$ Centro-Oeste e $1.5 \%$ Norte). Os participantes apresentavam diferentes níveis educacionais, sendo $59.4 \%$ estudantes de graduação, $16.9 \%$ era estudantes de pósgraduação, $12 \%$ relataram ter completado pósgraduação, $11 \%$ o ensino médio, e por fim $0,5 \%$ relataram possuir ensino médio incompleto).

A segunda amostra foi composta por 248 adolescentes, de ambos os sexos (50.9\% do sexo masculino), com idades entre 12 e 17 anos ( $\mathrm{M}=$ $13.93 \pm 1.39$ ), estudantes da rede privada de uma escola do interior de São Paulo.

\section{Instrumentos}

Questionário sociodemográfico. $\mathrm{O}$ questionário foi construído especificamente para presente pesquisa e é composto de questões para obtenção de informações de caráter descritivo referentes ao sexo, idade, escolaridade e região do país dos participantes.

Kessler Psychological Distress Scale (K10) (Escala de Distresse Psicológico de Kessler - K10)
Adaptada para o português brasileiro por Peixoto, Zanini e Andrade (2020), a K10 é uma escala de 10 itens que investiga a frequência de sintomas de distresse psicológico durante os últimos 30 dias. O instrumento de autorrelato é respondido em escala Likert de cinco pontos correspondentes a frequência de vivências estresse em que 1 indica "nenhuma vez" e 5 "todo o tempo. O instrumento apresenta bom índice de consistência interna $(\alpha=.87)$ e" baseando-se em perguntas sobre sintomas de ansiedade (ex. de item: Nos últimos 30 dias, com que frequência você se sentiu nervoso(a)?) e depressão (ex.: de item: Nos últimos 30 dias, com que frequência você se sentiu triste e que nada podia animálo(a)?). Estudos da versão brasileira da K10 indicaram adequação da estrutura bifactor composta por um fator geral (distresse global) e dois fatores específicos (depressão e ansiedade) (Peixoto, Zanini, \& Andrade, 2020).

- Benign and Malicious Envy Scale (BeMaS).

A escala tem como objetivo avaliar a inveja maliciosa e benigna por meio afirmações que expõem situações rotineiras de comparações sociais. $\mathrm{O}$ instrumento é composto por 10 itens (5 itens para cada um dos fatores), como por exemplo "Quando invejo outras pessoas, me concentro em como posso me tornar igualmente bem-sucedido(a) no futuro.", representando a inveja benigna e "Sinto rancor por pessoas que invejo." para a inveja maliciosa. com escala de resposta do tipo Likert de seis pontos. Baseandose na abordagem bidimensional, estrutura-se em dois fatores: inveja maliciosa e inveja benigna, os quais buscam discriminar as motivações que precedem determinados comportamentos, como crescimento pessoal e medo do fracasso. Vale ressaltar que estudos de adaptação da BeMaS para o português brasileiro foram realizados por meio do procedimento de tradução reversa e a estimativa de evidências de validade com base na estrutura interna indicaram adequação da estrutura fatorial e bons índices de precisão (Peixoto et al, no prelo).

- Dark Triad (Escala Tríade Sombria SD3Short).

A SD3-short originou-se da Dark Triad (Paulhus \& Williams, 2002), tendo sido utilizada para o presente estudo a versão adaptada para o contexto brasileiro por Hauck, Carvalho e Jonason 
(2015) que apresentou consistência interna de $\alpha=$.84. O instrumento avalia os traços da personalidade sombria: maquiavelismo, narcisismo e psicopatia por meio de 27 itens, sendo nove para cada dimensão, que são respondidos em escala Likert de cinco pontos. Como exemplo de item do fator de maquiavelismo: "Não é esperto contar os seus segredos", narcisismo "As pessoas me veem como um verdadeiro líder" e psicopatia "As pessoas dizem que eu sou descontrolado".

\section{Procedimentos}

\section{Procedimento ético e coleta de dados}

Após a aprovação do projeto de pesquisa pelo Comitê de Ética da Universidade de Pernambuco, sob o número 26318619.2.0000.5191, iniciou-se o processo de coleta de dados. Para isso, foi disponibilizado um link nas redes sociais para que os participantes pudessem responder $\mathrm{o}$ questionário, visto que a coleta ocorreu virtualmente, por meio da plataforma Google Forms. Aqueles que optaram por participar, deveriam assinar eletronicamente o Termo de Consentimento Livre Esclarecido (TCLE), concordando com a sua participação e afirmando ter mais de 18 anos. Após a concordância, os participantes adultos responderam o questionário sociodemográfico e Benign and Malicious Envy Scale (BeMaS). Uma parcela da amostra de adultos $(\mathrm{n}=289)$ respondeu, além da BeMaS, as seguintes escalas: Dark Triad e a Kessler Psychological Distress Scale - K10, nessa ordem, considerando que, para este estudo, foram realizadas mais de uma coleta em momentos distintos. Estimou-se uma duração média de 20 minutos.

Os participantes adolescentes, por sua vez, responderam ao questionário sociodemográfico e a BeMaS presencialmente, coletivamente, após consentimento dos responsáveis e assinatura do termo de assentimento. As aplicações foram realizadas em contexto escolar com duração média de 20 minutos.

\section{Análise de dados}

Considerando os objetivos propostos, buscouse realizar a análise dos dados por meio da Análise Fatorial Confirmatória (AFC) no software
MPlus versão 7.3 (Muthén \& Muthén, 2017), testando a adequação estrutural de dois fatores da BeMaS. Para tanto, empregou-se o método de estimação Weighted Least Squares Mean and Variance-adjusted (WLSMV) aplicado a matriz de correlação policórica, e tendo declarado os itens como variáveis ordinais. Para avaliação do modelo fatorial foram adotados os seguintes índices de ajuste: Comparative Fit Index (CFI), Tucker-Lewis Index (TLI), os quais foram classificados como adequados quando os valores obtidos fossem superiores a .90 e bons quando superiores a .95. Além disso, foi utilizado o Root Mean Square Error of Approximation (RMSEA) tendo como referências valores inferiores .10 como aceitáveis, entre .08 como e .05 , como adequados e menores que .05 como bons (Marsh, 2007).

Na sequência foi realizada a avaliação da invariância do modelo fatorial de medida entre grupos considerando a amostra de adolescentes e adultos, tendo sido empregada a Análise Fatorial Confirmatória Multigrupo (AFCMG) com estimação de modelo nos níveis configuracional, métrico e escalar. Foram considerados os mesmos índices de ajustes supracitados e novamente os itens foram declarados como ordinais. $\mathrm{O}$ pressuposto de invariância entre os grupos foi avaliado por meio da variabilidade do índice CFI e RMESA ( $\triangle \mathrm{CFI} \leq .01)$ (Cheung \& Resnvold, 2002).

Para avaliar os índices de fidedignidade do BeMaS utilizou-se os coeficientes alfa de Cronbach e Ômega de McDonald (McDonald, 1999). Segundo a literatura, foram adotados, para ambos os coeficientes, valores iguais ou superiores a .7, como bons indicadores de precisão (Tabachnick \& Fidell, 2018). Por fim, empregou-se índices de correlação de Pearson, para estimar a associação entre os indicadores de inveja e as outras variáveis externas empregadas na presente pesquisa (depressão, ansiedade, distresse psicológico, tríade sombria), considerouse como indicadores de significância estatística níveis de $p<.05$, e classificação das magnitudes de correlações entre 0 e .9 como nula, entre .10 e .29 como fraca, entre 30 e .49 moderado e superior a .50 como forte (Cohen, 1988). 


\section{Resultados}

Os resultados da AFC revelaram índices de ajuste classificados como adequados ao modelo de medida bidimensional da BeMaS para a amostra de adultos: $\quad x^{2}=139,173 ; \quad \mathrm{gl}=34 ; \quad p<.001 \quad x^{2} /$; CFI $=.954 ; \quad$ TLI $=.934 ; \quad$ RMSEA $=.087$ IC $90 \%$ 0,072-0,102. O modelo fatorial é apresentado na Figura 1, onde se verificam as cargas fatoriais e erro padrão associados a estimação destas cargas.

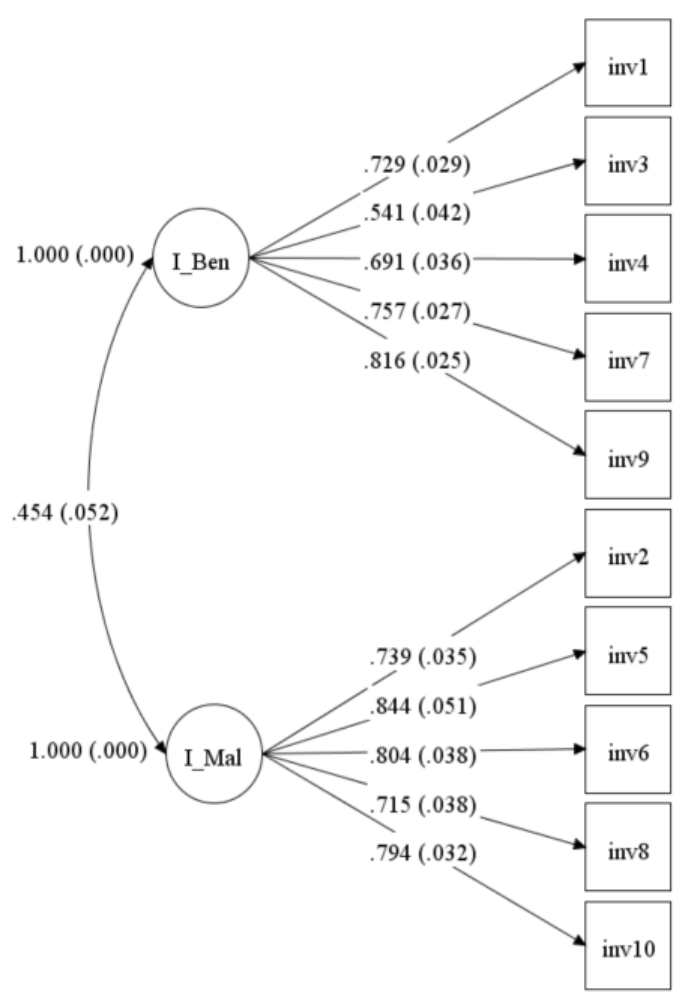

Figura 1. Modelo fatorial confirmatório BeMaS para adultos

O modelo fatorial exposto demonstra as cargas fatoriais apresentadas pelos itens em cada um dos fatores e, também, as correlações entre os fatores e o índice de confiabilidade. De acordo com a Figura 1, é possível verificar que o modelo testado, apontou significativas cargas fatoriais, com valores iguais ou superiores a .541, bem como baixos erros padrões associados às variáveis do modelo. Os resultados mostram que os itens apresentaram cargas fatoriais de acordo com o pressuposto teórico descrito pelo modelo bidimensional da inveja. Deste modo, os itens que compõem o fator Inveja Benigna (itens: 1, 3 4, 7 e 9) apresentaram cargas fatoriais na dimensão em questão, as quais variaram de .541 (item 3) a .816 (item 9). Enquanto, em relação ao fator inveja maliciosa, os itens $(2,5,6,8$ e 10) apresentaram cargas fatoriais, no respectivo fator, que variaram de .715 (item 8) a .844 (item 5). Quanto a correlação entre os fatores, foi obtido um índice de correlação moderado $(r=.454)$. Tais resultados permitem inferir evidências de validade baseadas na estrutura interna da BeMaS para a população adulta brasileira. Ademais, os índices de precisão apresentados para cada um dos fatores foram adequados, para o fator inveja benigna obtendo-se $\alpha=.839$ e $\omega=.840$, enquanto no fator inveja maliciosa observou-se $\alpha=.884$ e $\omega=.885$, dados que sustentam a fidedignidade da versão brasileira para adultos.

$\mathrm{Na}$ sequência, a invariância do modelo foi testada em função das faixas etárias dos participantes (adolescentes e adultos). Os resultados são apresentados no Quadro 1, na qual são expostos os índices de ajustes para os modelos configuracional (equivalência da estrutura fatorial geral e número de fatores), métrico (equivalência das cargas fatoriais dos itens) e escalar (equivalência dos níveis dos interceptos dos itens), dessa forma, partindo do menos restritivo para o mais restritivo.

A partir dos índices expostos no Quadro 1, é possível verificar que os resultados indicaram a equivalência do modelo fatorial nos diferentes níveis (Configuracional, métrico e escalar), sugerindo a equivalência do modelo bidimensional para avaliação de grupos compostos por adultos e adolescentes, considerando o fato de que os índices de ajustamento não foram prejudicados com a restrição dos modelos (valores de $\Delta \mathrm{CFI}$ entre os modelos são inferiores a .01).

Por fim, foram verificados os índices de correlação entre os escores apresentados nas variáveis inveja maliciosa e inveja benigna com as seguintes variáveis: depressão, ansiedade, distresse psicológico global, maquiavelismo, psicopatia e narcisismo. Para isso, Para isso, foi considerada a amostra de 289 participantes adultos, de ambos os sexos (idade 29.4 \pm 10.7 ; $72.7 \%$, mulheres) que responderam a todos os instrumentos (BeMas, Dark Triad, e K10). Os resultados estão expostos na Quadro 2. 
Quadro 1. Modelo de Invariância Multigrupos em função da fase de desenvolvimento dos participantes

\begin{tabular}{lcccccccc}
\hline Modelo & $\chi^{2}$ & $G l$ & CFI & TLI & RMSEA & IC90\% & CFI $(\Delta)$ & $\operatorname{RMSEA}(\Delta)$ \\
\hline Configuracional & 219.009 & 68 & .957 & .943 & .082 & $.070-.095$ & & \\
Métrico & 252.077 & 76 & .950 & .941 & .084 & $.073-.096$ & .007 & -.002 \\
Escalar & 102.499 & 114 & .936 & .949 & .078 & $.068-.087$ & .014 & .006 \\
\hline
\end{tabular}

Quadro 2. Matriz de Correlação entre BeMaS, K10 e Dark Triad (n=289)

\begin{tabular}{lcc}
\hline & $\begin{array}{c}\text { Inveja } \\
\text { Benigna }\end{array}$ & $\begin{array}{c}\text { Inveja } \\
\text { Maliciosa }\end{array}$ \\
\hline Maquiavelismo & $.353^{*}$ & $.411^{*}$ \\
Narcisismo & $.325^{*}$ & $.268^{*}$ \\
Psicopatia & $.235^{*}$ & $.451^{*}$ \\
Ansiedade & .091 & $.231^{*}$ \\
Depressão & .051 & $.277^{*}$ \\
Distresse & .070 & $.276^{*}$ \\
\hline Nota. ${ }^{*} p<.001 ;$ Distresse = Distresse psicológico global
\end{tabular}

Conforme observado no Quadro 2, os resultados demonstraram que a inveja maliciosa apresentou correlações positivas e moderadas com maquiavelismo $(r=.411)$ e psicopatia $(r=.451)$, bem como correlações positiva de intensidade fraca com narcisismo $(r=.268)$, ansiedade $(r=.231)$, depressão $(r=.277$, ) e distresse global $(r=.276)$. Por outro lado, a inveja benigna apresentou correlações positivas de magnitude moderada com os fatores da tríade sombria da personalidade, a saber: maquiavelismo $(r=.353)$; narcisismo ( $r=.325)$; e magnitude fraca com psicopatia $(r=.235)$, bem como correlação nula e não significativa com as variáveis ansiedade $(r=.091)$, depressão $(r=.051)$ e distresse global $(r=.070)$.

\section{Discussão}

A presente pesquisa teve como objetivo principal estimar as primeiras evidências de validade e precisão da BeMas para a população adulta brasileira. Para isso, empregou-se os procedimentos estatísticos AFC, AFCMG (Análise Fatorial Confirmatória Multigrupo), avaliação da precisão e associação com variáveis externas. Os resultados sugerem que o instrumento se caracteriza como uma medida adequada dos indicadores de inveja para essa parcela da população, com estrutura interna composta por dois fatores (inveja maliciosa e benigna), correspondentes a proposta teórica que deu base ao processo de desenvolvimento da escala (Lange \& Crusius, 2015).

Em relação a estrutura interna, os resultados observados podem ser considerados coerentes com aqueles obtidos no estudo que deu origem a BeMaS junto a adultos alemães, e falantes nativos de língua inglesa (Lange \& Crusius, 2015). Neste estudo, os autores observaram bons índices de ajuste dos dados ao modelo de medida composto por dois fatores: $\chi^{2}(34)=189.89, \quad \mathrm{GFI}=.96$, CFI=.97, AGFI=.93, e RMSEA=.07 CI95\%[.06.08]. Também apresentam semelhança aos resultados de estudos internacionais que adaptaram e estimaram as propriedades psicométricas da BeMas, por meio de AFC, para a avaliação de adultos estadunidenses $\chi^{2}(34)=$ 125.029, CFI=.97, e RMSEA $=.058$ CI90\%[.047$.069]$; russos $\chi^{2}(34)=174.183, \quad \mathrm{CFI}=.93, \quad \mathrm{e}$ RMSEA $=.076 \quad$ CI90\%[.065-.088], alemães $\chi^{2}(34)=131.087, \quad$ CFI $=.94, \quad$ e $\quad$ RMSEA $=.072$ CI90\%[.059-.085], e poloneses $\chi^{2}(33)=79.167$, CFI=.978, e RMSEA=.044 CI90\%[.032-.056]. Neste sentido, os resultados ligeiramente superiores para versão polonesa se explicam pela adição de um parâmetro no modelo, correlação entre os resíduos dos itens 6 e 8 (Kwiatkowska et al., 2020).

Em relação às cargas fatoriais apresentadas pelos itens nos seus respectivos fatores, os valores observados na presente pesquisa, superiores a .5 , indicam que os itens representam adequadamente as variáveis latentes alvo da avaliação (os respectivos fatores). Além disso, indicam que os itens não são explicados pelo outro fator $e$, portanto, o modelo não é penalizado mesmo com o estabelecimento de um modelo restrito, como na $\mathrm{AFC}$, em que a carga fatorial é estimada apenas no fator de origem (teoricamente) e fixada em zero em outros fatores (Franco \& Valentini, 2017). Outras evidências empíricas desta estrutura parcimoniosa da BeMaS foram observadas por meio de modelo exploratório (Peixoto et al., no prelo), em que os itens saturaram fortemente em 
seus fatores de origem $(\geq .588)$ e com cargas muito próximas de zero no outro fator $(\leq .1)$.

Ainda sob esta perspectiva, os resultados corroboram estudos de evidências de validade com base na estrutura fatorial estimados para outras versões da BeMaS por meio de modelos saturados, ou seja, modelos que permitem que todos os itens se relacionem com todos os fatores do modelo de medida (Marsh et al., 2020). Nesta direção, Kwiatkowsk et al. (2020) destacam o próprio estudo de Lange e Crusius (2015) em que demonstraram a pertinência do modelo composto por dois fatores por meio da AFE, bem como os estudos de Çırpan e Özdoğru (2017) e Sawada e Fujii (2016) juntos as versões turca e japonesa da BeMaS.

Adicionalmente, no contexto brasileiro Peixoto et al. (no prelo) demonstraram a adequação do modelo de dois fatores para BeMaS em uma amostra composta por adolescentes, utilizando diferentes métodos de retenção de fatores (Análise Paralela, Método Hull, Exploratory Grafh Analysis) e do agrupamento de itens coerentes com os fatores inveja benigna e inveja maliciosa via AFE. Dessa forma, pode-se afirmar que a partir dos resultados deste estudo foi possível confirmar o modelo teórico bidimensional da inveja para avaliação de adultos por meio da AFC, bem como a estimação de novas evidências de validade da versão brasileira da BeMas (AERA, APA \& NCME, 2014).

Por fim, destaca-se uma característica do índice de ajuste RMSEA que apresentou valor no limite de valores classificados como adequados $\leq .08$ ou valores apenas aceitáveis $\leq .1$ (Marsh, 2007), ressalta-se que valores semelhantes também foram observados nos estudos internacionais supracitados. Contudo, uma importante explicação para tal fenômeno diz respeito a própria natureza deste índice de ajuste, que corresponde à média dos quadrados dos erros de aproximação e, portanto, estimado com base no tamanho da amostra, parâmetro de não centralidade, valores de qui-quadrado e graus de liberdades do modelo alvo. Nesse sentido, Kenny et al. (2014) demonstraram por meio de uma revisão de estudos teóricos e de simulação que o valor de RMSEA pode ser prejudicado em modelos que contam com baixo grau de liberdade. Desta forma, sugere-se que este pode ser um dos motivos que contribuíram para estimativa destoante, embora aceitável, para o valor de RMSEA.

Em relação aos indicadores de precisão estimados na presente pesquisa, observa-se que os índices de consistência interna superiores a 0,8 , por meio do alfa de Cronbach, são semelhantes aos observados em pesquisas com outras versões da BeMaS. Na versão original foram observados alfa de Cronbach entre .79 e .90 para o fator inveja benigna e .83 e .91 para o fator inveja maliciosa (Lange \& Crusius, 2015). Assim como para a versão espanhola, obtiveram um coeficiente geral de alfa de Cronbach de 85 (Navarro-Carrillo et al., 2017), enquanto nas pesquisas com amostras compostas pelo público americano, alemão, russo e polonês os alfas para inveja benigna variaram entre .83 e .88 e maliciosa entre .80 a .89 (Kwiatkowska et al., 2020).

Foram estimadas associações entre os fatores da BeMaS e outras variáveis externas. Os coeficientes de correlação indicaram associações positivas entre a inveja maliciosa, depressão, ansiedade, distresse psicológico. Esses resultados confirmam a hipótese de que experiência de níveis elevados de inveja maliciosa (caracterizada pela sentimentos de emoções negativas e hostilidade contra pessoas que apresentam características de superioridade ou posses não obtidas pelo sujeito invejoso), está associada a prejuízos nestes indicadores de saúde mental (Peixoto et al., no prelo). Nesta direção, Lange, Weidman \& Crusius (2018) sugerem que essa percepção de inferioridade e de impossibilidade de alcance dessas características ou posses podem fomentar comportamentos agressivos, sentimentos angústia psicológica e depressão.

Por outro lado, observou-se ausência de associação entre o fator inveja benigna e os indicadores de depressão, ansiedade, distresse psicológicos. Tais resultados são coerentes com a proposta teórica que embora sugira que a inveja benigna represente uma percepção de inferioridade, nessa forma de comparação social há uma tendência do sujeito em se esforçar para superar a própria condição de inferioridade e melhorar o status pessoal (Lange, Blatz \& Crusius, 2017; Sawada \& Fujii, 2016).

Sob esta perspectiva ainda, destaca-se o estudo de Peixoto et al (no prelo). Os autores 
deste estudo apresentam resultados indicativos de que a inveja benigna se relacionava positivamente com a variável satisfação com a vida e também não apresentava associações significativas com os indicadores de depressão, estresse, ansiedade, e angústia psicológica, avaliados por meio da Escala de Depressão, Ansiedade e Estresse (DASS-21).

Em relação a associação entre a BeMaS e os indicadores psicopatológicos: maquiavelismo, narcisismo e psicopatia (Jones \& Paulhus, 2014), verificou-se que os indicadores se associavam positivamente com a tríade sombria da personalidade, embora a associação entre psicopatia com a inveja benigna tenha sido de baixa magnitude. Resultados semelhantes foram obtidos por Lange, Paulhus e Crusius (2017) o que levou os autores contraporem a expectativa teórica inicial de que a inveja benigna se associaria apenas a fenômenos psicológicos positivos (Lange \& Crusius, 2015).

Em relação a inveja maliciosa, as correlações moderadas com maquiavelismo e psicopatia, bem como de baixa magnitude com narcisismo corroboram a hipótese teórica e os resultados apresentados por Lange, Paulhus e Crusius (2017). É esperado que a vivência da inveja maliciosa resulte sentimentos de afeto negativos e em estratégias e comportamentos maquiavélicos, como sabotagem e investimentos para destruição da imagem do sujeito invejado (Lange et al., 2016). Neste caso, a associação com a psicopatia representa a ligação entre inveja maligna e os comportamentos impulsivos e irresponsáveis contra o sujeito invejado, como agressão e tentativas de destruição do outro (Lange, Paulhus \& Crusius, 2017). Os autores destacam a importância destes construtos em outros contextos, como o esportivo por exemplo, em que agressões físicas e verbais podem ser direcionadas a um adversário, o qual é invejado por suas habilidades esportivas ou competências físicas.

Por fim, a associação de magnitude fraca entre inveja maliciosa e narcisismo também são coerentes com os estudos supra citados, e sugere que pessoas narcisistas se engajam em comparações sociais para comprovarem o quão bem sucedidos são em relação aos outros. Essa magnitude fraca pode ser explicada, uma vez que denota uma característica egocêntrica e necessidade exacerbada de admiração inerente ao narcisismo (Pereira \& Paixão, 2019; Pechorro et al, 2019) e não necessariamente numa tentativa de destruição do objeto desta comparação. Ainda nessa compreensão, também faz sentido teórico a observação de maior magnitude de associação desta variável com a inveja benigna, haja vista a expectativa de sucesso a ser alcançado tanto na autoadmiração narcisista quanto na inveja benigna (Lange, Crusius, \& Hagemeyer, 2016).

De posse de evidências de validade baseadas na estrutura interna, relação com outras variáveis e precisão para a versão brasileira da BeMaS em uma amostra de adultos, buscou-se, também, estimar evidências de invariância do modelo de medida em função da fase de desenvolvimento dos respondentes (adolescentes e adultos). A necessidade de estudos desta natureza corresponde ao fato de que este é o primeiro passo para a comparação de grupos assegurando-se de que eles são avaliados de forma equivalente pelo instrumento (Chen, 2007), e neste caso para a fundamentação de futuros estudos que investiguem aspectos desenvolvimentais associados ao construto da inveja.

Os resultados sugerem que o instrumento apresenta invariância forte, ou seja, equivalência da estrutura fatorial (modelo configuracional), das cargas fatoriais (modelo métrico) e dos intercepto dos itens (modelo escalar), quando controlado o nível de traço latente dos respondentes, sugerindo a adequação do emprego da BeMaS em estudos que tenham por objetivo comparar os escores destes estratos amostrais. Vale destacar que o índice CFI indicou variabilidade $(\Delta=.014)$ entre o modelo métrico e escalar, sugerindo a não invariância do modelo escalar, contudo a estatística RMSEA $(\Delta=.006)$ sustentava a estabilidade do modelo mesmo com adição das novas restrições. De acordo com Chen (2007) a estimativa da invariância é uma técnica complexa e, portanto, deve ser avaliada por diferentes índices, mais especificamente o autor sugere que em amostras com número de participantes superior a 300, no teste de invariância do intercepto, degradações no índice CFI $\geq-.010$, complementadas por mudanças $\geq .015$ em RMSEA indicariam não invariância do modelo. Considerando que a mudança observada no CFI da presente pesquisa foi positiva, observou-se a 
melhora do índice com as restrições no modelo, complementada pelo baixo valor de $\Delta$ no índice RMSEA, pode-se concluir a invariância do modelo de medida entre os grupos estudados.

\section{Considerações finais}

Pode-se afirmar que os objetivos da presente pesquisa foram satisfatoriamente alcançados, e que a versão brasileira da BeMaS apresenta evidências de validade e precisão na avaliação de adultos. Compreende-se que tais esforços são importantes para o avanço dos estudos sobre a inveja no contexto brasileiro, uma vez que o instrumento operacionaliza uma compreensão atual deste construto a partir de uma perspectiva multidimensional. Porém, ressalta-se a importância de realização de novos estudos que sejam capazes de verificar a invariância da escala em relação ao sexo.

Sugere-se ainda que o instrumento seja empregado em pesquisas que contem com maior equilíbrio em relação ao sexo dos participantes permitindo estimativa de invariância entre esses grupos. Ainda como limitação, aponta-se as características da amostra não probabilística, representada majoritariamente por residentes da região sudeste do Brasil, o que exige cautela na generalização dos resultados para outras zonas do país.

\section{Referências}

American Educational Research Association, American Psychological Association, \& National Council on Measurement in Education (2014). Standards for educational and psychological testing. Washington: American Educational Research Association.

Çırpan, Y., \& Özdoğru, A. (2017). Turkish adaptation of BeMaS Benign and Malicious Envy Scale: Transliteral equivalence, reliability and validity study. Anatolian Journal of Psychiatry. https://doi.org/18.1.10.5455/apd.256664

Chen, F. (2007). Sensitivity of goodness of fit indexes to lack of measurement invariance. Structural Equation Modeling, 14, 464-504. https://doi.org/10.1080/10705510701301834.
Cohen, J. (1988) Statistical Power Analysis for the Behavioral Sciences, 2nd ed. Hillsdale, $\mathrm{NJ}$ :

Erlbaum.Franco, V. R., Valentini, F., \& Iglesias, F. (2017). Introdução à análise fatorial confirmatória. Em B. F. Damásio \& J. C. Borsa. (Org.), Manual de Desenvolvimento de Instrumentos Psicológicos (pp. 295-322). Vetor.

Hauck, N., Filho, Carvalho, L. F., \& Jonason, P. K. (2015). Análise fatorial confirmatória da versão em português brasileiro da escala Dirty Dozen. Mesa redonda apresentada no VII Congresso Brasileiro de Avaliação Psicológica.

Recuperado em <http://www.ibapnet.org.br/congresso201 5/anais/listaresumos.htm>

Jones, D. N., \& Paulhus, D. L. (2014). Introducing the Short Dark Triad (SD3): A brief measure of dark personality traits. Assessment, 21, 28-41. https://doi.org/10.1177/1073191113514105

Kenny, D. A., Kaniskan, B., \& McCoach, D. B. (2014). The Performance of RMSEA in Models With Small Degrees of Freedom. Sociological Methods \& Research, 44(3), 486-507.

https://doi.org.10.1177/0049124114543236

Kwiatkowska, M. M., Rogoza, R., \& Volkodav, T. (2020). Psychometric properties of the Benign and Malicious Envy Scale: Assessment of structure, reliability, and measurement invariance across the United States, Germany, Russia, and Poland. Psychometric Properties of the Bemas, 0-2.

Lange, J., Blatz, L., \& Crusius, J. (2017). Dispositional envy: A conceptual review. In V. Zeigler-Hill \& T. K. Shackelford (Eds.), The SAGE handbook of personality and individual differences: Applications of personality and individual differences ( $\mathrm{p}$ 424-440). Sage Reference. https://doi.org/10.4135/978152645 1248.n18

Lange, J., \& Crusius, J. (2015). Dispositional envy revisited: Unraveling the motivational dynamics of benign and malicious envy. Personality and Social Psychology Bulletin, 41(2), 284-294 https://doi.org/10.1177/0146167214564959 
Lange, J., Crusius, J., \& Hagemeyer, B. (2016). The Evil Queen's dilemma: Narcissistic admiration and rivalry to benign and malicious envy. European Journal of Personality, 30, 168-188. https://doi.org/10.1002/per.2047

Lange, J., Paulhus, D. L., \& Crusius, J. (2018). Elucidating the dark side of envy: Distinctive links of benign and malicious envy with dark personalities. Personality and Social Psychology Bulletin, 44(4), 601-614. https://doi.org/10.1177/0146167217746340

Lange, J., Weidman, A., \& Crusius, J. (2018). The painful duality of envy: Evidence for an integrative theory and a meta-analysis on the relation of envy and Schadenfreude. Journal of Personality and Social Psychology. https://doi.org/114.10.1037/pspi0000118.

Marôco, J. (2010). Análise de equações estruturais: Fundamentos teóricos, software \& aplicações. ReportNumber, Lda.

Marsh, H. W., Guo, J., Dicke, T., Parker, P. D., \& Craven, R. G. (2020). Confirmatory factor analysis (CFA), exploratory structural equation modeling (ESEM), and set-ESEM: optimal balance between goodness of fit and parsimony. Multivariate behavioral research, 55(1), 102-119. https://doi.org/10.1080/00273171.2019.1602503

Marsh, H. W. (2007). Application of confirmatory factor analysis and structural equation modeling in sport and exercise psychology. In G. Tenenbaum \& R. C. Eklund (Eds.), Handbook of Sport Psychology (3rd ed., pp. 774-798).

McDonald, R. P. (1999). Test theory: A unified treatment. Mahwah, NJ: Lawrence Erlbaum.

Milfont, T. L., \& Gouveia, V. V. (2009). A capital sin: Dispositional envy and its relations to wellbeing. Interamerican Journal of Psychology, 43(3), 547-551.

Muthén, L. K., \& Muthén, B. O. (2017). Mplus User's Guide. Seventh Edition.

Navarro-Carrillo, G., Beltrán-Morillas, A., ValorSegura, I., \& Expósito, F. (2017): What is behind envy? Approach from a psychosocial perspective /¿Qué se esconde detrás de la envidia? Aproximación desde una perspectiva psicosocial. Revista de Psicología Social. https://doi.org/10.1080/02134748.2017.1297354
Paulhus, D. L., \& Williams, K. M. (2002). The dark triad of personality: Narcissism, machiavellianism, and psychopathy. Journal of Research in Personality, 36(6), 556-563. https://doi.org/10.1016/S00926566(02)00505-6

Parrott, W. G. (1991). The emotional experiences of envy and jealousy. In P. Salovey (Ed.), The psychology of jealousy and envy (pp. 3-30). New York: Guilford.

Pechorro, P., Nunes, C., Gonçalves, R. A., \& Simões, M. R. (2019). Estudo de Validação do Inventário de Personalidade Narcísica - 13 numa Amostra Escolar de Jovens Portugueses. Revista Iberoamericana de Diagnóstico y Evaluación - e Avaliação Psicológica, 50(1). https://doi.org/10.21865/RIDEP50.1.06

Peixoto, E. M., Campos, C. R., Oliveira K. S., Tartaro, G. K., \& Baptista, M. N. (no prelo). Benign and Malicious Envy Scale: Adaptação transcultural e evidências de validade para adolescentes. Psicologia: Teoria e Prática.

Peixoto, E. M., Zanini, D. S., Andrade, J. M. (2020). Kessler Distress Scale (K10): an application of the Rating Scale Model (Manuscrito submetido).

Pereira, C., \& Paixão, R. (2019). Estrutura Fatorial da Versão Portuguesa da Escala de Narcisismo Hipersensível. Revista Iberoamericana de Diagnóstico y Evaluación - e Avaliação Psicológica, 53(4). https://doi.org/10.21865/ridep53.4.02

Sawada, M., \& Fujii, T. (2016). Do envious people show better performance?: Focusing on the function of benign envy as personality trait. The Japanese Journal of Psychology, 87(2), 198204. https://doi.org/10.4992/jjpsy.87.15316

Smith, R. H., \& Kim, S. H. (2007). Comprehending envy. Psychological Bulletin, 133(1), 46-64. https://doi.org/10.1037/0033-2909.133.1.46

Tabachnick, G. B., \& Fidell, L. S. (2019). Principal Components and Factor Analysis. In G. B. Tabachnick \& L. S. Fidell (Eds.), Using Multivariate Statistics (pp. 476-527). Pearson.

Van de Ven, N., Zeelenberg, M., \& Pieters, R. (2009). Leveling up and down: The experiences of benign and malicious envy. Emotion, 9(3), 419-429. https://doi.org/10.1037/a0015669 